INTERVENTIONAL CARDIOLOGY AND SURGERY

\title{
Findings of intravascular ultrasound during acute stent thrombosis
}

\author{
F Alfonso, A Suárez, D J Angiolillo, M Sabaté, J Escaned, R Moreno, R Hernández, C Bañuelos, \\ C Macaya
}

Heart 2004;90:1455-1459. doi: 10.1136/hrt.2003.026047

See end of article for authors' affiliations .....................

Correspondence to: Dr F Alfonso, Cardiología Intervencionista, Instituto Cardiovascular, Hospital Universitario Clínico "San Carlos", Ciudad Universitaria, Plaza Cristo Rey, Madrid 28040, Spain; falf@hotmail.com

Accepted

14 February 2004 intravascular ultrasound (IVUS) in evaluating patients Objective: To evaluate the potential role of intravascular ultrasound (IVUS) in evaluating patients
experiencing an episode of acute stent thrombosis. Design and setting: Prospective observational study in a cardiac catheterisation laboratory in a university teaching hospital.

Patients and interventions: IVUS was used to examine 12 patients undergoing coronary interventions for stent thrombosis to gain further mechanistic insights and to guide treatment. IVUS studies were obtained before and after intervention with a motorised pullback device.

Main outcome measures: Qualitative and volumetric IVUS analyses.

Results: Angiographically, 10 patients had occluded vessels and two patients had intraluminal filling defects within the stent. IVUS showed an occlusive thrombus in all patients. Thrombus volume was 90 (77) $\mathrm{mm}^{3}$, which was 51 (21)\% of total stent volume. There was evidence of severe stent underexpansion in most patients and no patient fulfilled standard criteria for optimal stent implantation. Stent malapposition was detected in four patients, edge dissections were seen in two patients, and significant inflow-outflow disease was present in 11 patients. During interventions IVUS findings led to the use of higher pressures or larger balloons than those used during initial stenting in 10 patients. In addition, four patients required additional stenting, whereas a thrombectomy device alone was selected for one patient. After the procedure final minimum stent area $\left(7.1(2.1) \vee 5.3(2) \mathrm{mm}^{2}, \mathrm{p}<0.005\right)$ and stent expansion (83.2 (17) v 62.1 (15)\%, $\mathrm{p}<0.005)$ improved compared with pre-interventional values. However, residual lining thrombus was still visualised in eight patients $\left(25(19) \mathrm{mm}^{3}\right.$, accounting for a $17 \%$ of final stent volume).

Conclusions: IVUS provides an attractive technique to characterise fully the pattern of stent thrombosis, to identify readily the underlying mechanical predisposing factors, and to guide repeated coronary interventions.
A cute stent thrombosis remains an infrequent but devastating complication in patients undergoing coronary stenting. The occurrence of stent thrombosis in the current era of high pressure stent implantation and combined antiplatelet treatment ranges from $1 \%$ to $2 \% .{ }^{1-3}$ Most patients with this complication eventually develop a myocardial infarction despite urgent management with repeated coronary interventions or thrombolysis. ${ }^{1-3}$ Accordingly, further insights into predisposing factors, mechanisms, and management of these patients are warranted.

Intravascular ultrasound (IVUS) has been largely used to optimise stent deployment ${ }^{1-5}$ but its use in patients presenting with acute stent thrombosis has not been previously reported. In this prospective study we sought to determine the value of IVUS for patients experiencing an episode of stent thrombosis.

\section{METHODS}

\section{Patient selection}

Fifty consecutive patients with a clinical suspicious of stent thrombosis, subsequently confirmed by angiography, were enrolled in the study from February 1999 to March 2003. Thus, the incidence of stent thrombosis during the study period was $1.2 \%$ (4116 patients treated with bare metal stents). Of these, 38 patients were excluded for logistical reasons, severe haemodynamic derangement, persisting angina with ECG changes (after crossing the stent with the guidewire), or failure to recanalise the vessel. In the remaining 12 patients, the thrombosed stent was studied by IVUS before intervention.

\section{Coronary interventions}

Several angiographic views were obtained after administration of intracoronary glyceryl trinitrate. Classic angiographic criteria were used for the diagnosis of thrombus and dissections. ${ }^{67}$ IVUS studies were performed, once the guidewire had successfully crossed the thrombosed stent, when a TIMI (thrombolysis in myocardial infarction) flow grade $\geqslant 2$ was obtained or, in the presence of TIMI l flow, if the patient was asymptomatic or chest pain had improved and ST segment changes were no longer present. However, in patients with ongoing chest pain and significant ST changes the protocol allowed quickly crossing the occluded stent with a deflated balloon catheter to see whether this manoeuvre would result in pain relief and TIMI grade $\geqslant 2$. This was considered to be a pragmatic approach considering that the crossing profile of current balloon catheters is smaller than that of the imaging catheters. Two patients entered the study after this minor mechanical intervention.

IVUS catheters with 30 or $40 \mathrm{MHz}$ mechanical transducers (Atlantis or Ultracross, Boston Scientific Corporation, Sunnyvale, California, USA) were used with a motorised pullback device at a constant speed $(0.5 \mathrm{~mm} / \mathrm{s})$. The imaging sequence started $1 \mathrm{~cm}$ distal to the stent and continued interruptedly up to the aortocoronary junction or at least 
Table 1 Clinical and angiographic features at initial stenting and during stent thrombosis

\begin{tabular}{|c|c|c|c|c|c|c|c|c|c|c|c|c|c|c|}
\hline \multirow[b]{2}{*}{ Patient } & \multirow[b]{2}{*}{$\begin{array}{l}\text { Age } \\
\text { (years) }\end{array}$} & \multirow[b]{2}{*}{ Sex } & \multirow[b]{2}{*}{$\begin{array}{l}\text { Vessel/ } \\
\text { symptoms* }\end{array}$} & \multicolumn{2}{|c|}{$\begin{array}{l}\text { QCA before } \\
\text { stenting }\end{array}$} & \multicolumn{2}{|c|}{$\begin{array}{l}\text { QCA after } \\
\text { stenting }\end{array}$} & \multirow{2}{*}{$\begin{array}{l}\text { Time } \\
\text { (stent to } \\
\text { occlusion) } \\
\text { (h) }\end{array}$} & \multirow{2}{*}{$\begin{array}{l}\text { Time (pain to } \\
\text { angiography) } \\
\text { (min) }\end{array}$} & \multirow{2}{*}{$\begin{array}{l}\text { Stent occlusion } \\
\text { presentation† }\end{array}$} & \multirow[b]{2}{*}{ Findings } & \multicolumn{2}{|c|}{ Final QCA } & \multirow[b]{2}{*}{ Outcome } \\
\hline & & & & $\begin{array}{l}\text { MLD } \\
(\mathrm{mm})\end{array}$ & \%DS & $\begin{array}{l}\text { MLD } \\
(\mathrm{mm})\end{array}$ & \%DS & & & & & $\begin{array}{l}\text { MLD } \\
(\mathrm{mm})\end{array}$ & \%DS & \\
\hline 1 & 39 & $M$ & LAD/UA & 0.6 & $82 \%$ & 1.9 & $12 \%$ & 0.5 & 15 & UA & IFD, TIMI 2 & 2.1 & $12 \%$ & Uneventful \\
\hline 2 & 50 & $M$ & $\mathrm{LAD} / \mathrm{MI}$ & 1.0 & $62 \%$ & 2.6 & $18 \%$ & 23 & 60 & STSE & TIMI 0 & 4.2 & $7 \%$ & Uneventful \\
\hline 3 & 68 & $\mathrm{~F}$ & LAD/MI & NA & NA & NA & NA & 1 & 60 & VF, STSE & TIMI 0 & 2.0 & $11 \%$ & NQWMI \\
\hline 4 & 68 & $M$ & LAD/UA & 0.6 & $77 \%$ & 2.0 & $31 \%$ & 1 & 45 & STSE & IFD, TIMI 1 & 2.1 & $10 \%$ & QWMI \\
\hline 5 & 56 & $M$ & LAD/MI & 0.9 & $55 \%$ & 1.8 & $10 \%$ & 0.3 & 105 & STSE & TIMI 0 & 2.4 & $13 \%$ & NQWMI \\
\hline 6 & 68 & $M$ & LCX/UA & 1.2 & $53 \%$ & 2.3 & $12 \%$ & 1 & 90 & STSE & TIMI 0 & 2.3 & $15 \%$ & QWMI \\
\hline 7 & 35 & M & LAD/MI & 0.6 & $72 \%$ & 2.3 & $15 \%$ & 96 & 120 & STSE & TIMI 0 & 2.6 & $14 \%$ & NQWMI \\
\hline 8 & 51 & $\mathrm{~F}$ & RCA/UA & 0.8 & $63 \%$ & 2.0 & $6.0 \%$ & 120 & 120 & STSE & TIMI 0, Ds & 2.1 & $20 \%$ & QWMI \\
\hline 9 & 54 & $M$ & LAD/UA & 0.9 & $63 \%$ & 2.2 & $14 \%$ & 672 & 130 & STSE & TIMI 0 & 2.4 & $11 \%$ & QWMI \\
\hline 10 & 64 & M & $\mathrm{LAD} / \mathrm{MI}$ & 0 & $100 \%$ & 1.8 & $26 \%$ & 96 & 180 & STSE & TIMI 0 & 2.3 & $12 \%$ & NQWMI \\
\hline 11 & 63 & M & $\mathrm{RCA} / \mathrm{MI}$ & 0 & $100 \%$ & 2.8 & $21 \%$ & 456 & 360 & STSE & IFD, TIMI 2 & 3.0 & $9 \%$ & NQWMI \\
\hline 12 & 64 & M & LAD/MI & 1.3 & $49 \%$ & 1.8 & $35 \% \S$ & 20 & 30 & STSE & TIMI 0 & 2.6 & $13 \%$ & NQWMI \\
\hline $\begin{array}{l}\text { \%DS, pe } \\
\text { coronar } \\
\text { quantita } \\
\text { myocarc } \\
{ }^{*} \text { Clinica } \\
\text { after the }\end{array}$ & $\begin{array}{l}\text { dial infar } \\
\text { ative coro } \\
\text { diamptor }\end{array}$ & $\begin{array}{l}\text { arction; } \\
\text { oms at } \\
\text { dure to }\end{array}$ & $\begin{array}{l}\text { angiography; } \\
\text { UA, unstable } \\
\text { initial stenting } \\
\text { avoid losing }\end{array}$ & $\begin{array}{l}\text { angina } \\
\text { tclinicc }\end{array}$ & $\begin{array}{l}\text { VF, ven } \\
\text { I present } \\
\text { diagona }\end{array}$ & tricular & $\begin{array}{l}\text { fibrillatic } \\
\text { stent thr }\end{array}$ & $\begin{array}{l}\text { ction; RCA, } \\
\text { om. } \\
\text { ombosis; †o }\end{array}$ & ht coronary arte & ase; §angiographi & c underexpan & $\begin{array}{l}\text { n ECG; } \\
\text { on of th }\end{array}$ & TIMI, $t$ & $\begin{array}{l}\text { ft circumflex } \\
\text { tion; QCA, } \\
\text { ombolysis in } \\
\text { as accepted }\end{array}$ \\
\hline
\end{tabular}

until a previously identified angiographically normal segment proximal to the stent was visualised. Then, manual advancement of the IVUS catheter was allowed to further interrogate the region of interest (saline flushing was discretional) but care was always taken to ensure that the total imaging time was kept to a minimum. In particular, once coronary flow was re-established saline flushing was required in selected cases to improve identification of residual thrombus or to confirm stent malapposition. All IVUS studies were recorded on 0.5 inch high resolution s-VHS tape for subsequent offline analysis. $^{8-10}$

\section{Angiographic and IVUS analyses}

Quantitative angiography was performed off line by experienced personnel, firstly, before and after initial stenting and, secondly, after treatment of the stent thrombosis with a validated system with an automatic edge detection algorithm (MEDIS, CMS 4.0, Leiden, the Netherlands). ${ }^{6}{ }^{10}$

Several quantitative and qualitative IVUS parameters were analysed as previously described. ${ }^{8-10}$ A validated system for volumetric IVUS analysis (EchoScan, TomTec, Unterchleissheim, Germany) was used for offline three dimensional reconstruction and volumetric analysis. The program constructs two longitudinal sections from the data set and identifies the contours corresponding to the lumen intima and the stent boundaries. The axial location of an individual planar image is indicated by a cursor, which can be used to scroll through the entire set of planar images. Subsequently, corrections may be made interactively by forcing the contour through visually identified points, which update the entire data set. Lumen and stent areas were measured every $0.16 \mathrm{~mm}$ of stent length. Thrombus volume was calculated as stent volume minus lumen volume. The reference segments were selected as the most normal looking segments on IVUS within $10 \mathrm{~mm}$ proximal and distal to the stent edges. ${ }^{8}$ Stent underexpansion was defined as minimum stent area $<80 \%$ of the mean proximal and distal reference areas. ${ }^{3}$ In addition, the MUSIC criteria for expansion ${ }^{4}$ were evaluated-namely, minimum stent area is (a) $>90 \%$ of the average reference lumen areas; (b) $>100 \%$ of the lowest reference lumen area; and (c) $>80 \%$ (average) and $>90 \%$ (lowest) of the reference segment lumen areas in cases of absolute minimum stent area $>9 \mathrm{~mm}^{2}$. Inflow-outflow disease was considered in the presence of plaque area $>50 \%$ of the external elastic lamina area.
In addition all IVUS studies were carefully reviewed jointly by two experienced observes for the evaluation of qualitative findings. Residual dissections or edge tears were described as flaps outside the stent edges with underlying blood speckling. ${ }^{31}$ An echo-free space with blood speckling between the stent struts and the vessel wall was diagnostic of malapposition. $^{3}$ These findings required confirmation on subsequent IVUS imaging once coronary flow had been re-established. Any material visualised within the stent, reducing its lumen, was considered to be thrombus after its configuration, echogenicity, and scintillating pattern were analysed. ${ }^{3}$

\section{Statistical analysis}

Categorical data are presented as values and percentages. Continuous variables (mean (SD)) were compared by the paired $t$ test. A p $<0.05$ was considered significant.

\section{RESULTS}

Table 1 summarises baseline clinical and angiographic characteristics of the study patients. The indication for initial stent implantation was always an acute coronary syndrome. Initial stenting was successful in all patients, which had good angiographic results on visual assessment. However, a suboptimal angiographic result was accepted for one patient in whom the stent encompassed a major side branch with ostial disease. Table 1 also presents findings of quantitative coronary angiography before and after initial stenting.

At the time of stent thrombosis all patients presented with prolonged chest pain and persisting ST segment changes within one month (20 minutes to 28 days) of initial stent implantation. In one patient antiplatelet treatment was withheld before elective non-cardiac surgery. The remaining 11 patients were taking standard dual antiplatelet drugs. On angiography 10 patients had occluded vessels (TIMI flow grade $0-1)$ despite the administration of coronary glyceryl trinitrate, whereas two patients with TIMI 2 flow had intraluminal filling defects within the stent.

IVUS was performed without complications (imaging time 209 (92) seconds) in all patients. Table 2 presents the main IVUS findings. An occlusive thrombus was detected in every patient (fig 1). Severe stent underexpansion was present in most patients and none of then fulfilled the MUSIC criteria for optimal stent implantation (table 2). Stent malapposition was clearly visualised in four patients. Another patient had a striking protrusion into the left main stem of a stent 
implanted in the left anterior descending artery, partially entrapping the circumflex coronary artery. In addition, edge dissections were seen in two patients and 11 patients had significant inflow-outflow disease according to the study criteria (fig 1). All these abnormal findings (except for one patient with residual dissection and another patient with outflow disease) were not detected by angiography. Thrombus volume was 90 (77) $\mathrm{mm}^{3}$ which was $51(21) \%$ of the total stent volume.

Balloon angioplasty was performed in 11 patients (one with adjunctive thrombectomy), and four patients required additional stenting (proximal or distal to the previous stent). In 10 of these 11 patients IVUS findings led to the use of higher pressures (seven patients) or larger balloons (five patients) than those used during initial stenting. A thrombectomy device alone was used in one patient. IVUS studies after the procedure were performed in 11 patients (table 3 ). Residual lining thrombus was visualised in eight of them $(25$ (19) $\mathrm{mm}^{3}$, accounting for a $17 \%$ of final stent volume). The degree of stent expansion clearly improved in these patients. Final minimum lumen diameter $(2.9(0.4) v 2.5(0.5) \mathrm{mm}$, $\mathrm{p}<0.001)$, minimum stent area $\left(7.1(2.1)\right.$ v $5.3(2) \mathrm{mm}^{2}$, $\mathrm{p}<0.005)$, and stent expansion (83.2 (17) v 62.1 (15)\%, $\mathrm{p}<0.005)$ improved compared with preinterventional values. However, four patients still did not fulfil the MUSIC criteria. Data consistent with transient distal embolisation during the procedure were detected in five patients (two transient no reflow, three minor downstream filling defects). Eventually, all patients ended the procedure with angiographic success and TIMI 3 flow. Ten patients received glycoprotein IIb/IIIa platelet receptor inhibitors during or immediately after the repeated intervention. Ten patients sustained a myocardial infarction (creatine kinase peak 949 (401) U): four $Q$ wave and six non-Q wave (table 1 ). Repeated procedures successfully treated recurrent stent thrombosis in three patients. Severe thrombocytosis was found in one of these patients and two had resistance to platelet inhibition as shown by a platelet function analyser (PFA-100, Dade, Behring, Miami, Florida, USA). No patient died during hospitalisation.

\section{DISCUSSION}

Coronary stenting is the dominant strategy in interventional cardiology and stent thrombosis remains the most feared early complication in patients undergoing these procedures. ${ }^{1-3}$ Even though the combination of aspirin and thienopyridines has gained a widespread reputation for the prevention of stent thrombosis, this dreaded complication still occurs in $1 \%$ to $2 \%$ of patients and, despite urgent adequate management, it is associated with an incidence of myocardial infarction up to $67 \%$ and an in-hospital mortality up to $15 \% .{ }^{1-4}$

IVUS has been a very useful tool to evaluate the results of stent implantation and, in fact, this technique provided the rationale for a shift in the implantation techniques leading to current standards. ${ }^{1-5}$ However, the potential value of IVUS for the management of patients with acute stent thrombosis has not been established. In a large retrospective multicentre registry Uren et al investigated whether IVUS provided additional information regarding prediction of stent thrombosis. Fifty three patients, who experienced stent thrombosis after stent implantation under IVUS guidance, were enrolled. Overall, $94 \%$ of patients had at least one abnormal IVUS finding (underexpansion, malapposition, inflow/outflow disease, dissection, or thrombus) at the end of the procedure, whereas angiographic abnormalities were detected in only $32 \%$ of patients $(p<0.001)$. These investigators concluded that, compared with angiography, IVUS features were abnormal at the time of deployment in the vast majority of stents associated with subsequent thrombosis. In a more recent study, ${ }^{13} 21$ patients with acute stent thrombosis were identified from a series of 7484 consecutive patients undergoing IVUS guided coronary stenting. Postinterventional IVUS results of lesions that subsequently experienced subacute stent thrombosis were compared with a matched cohort of lesions with an uneventful outcome. IVUS identified at least one cause for abrupt closure (dissection, thrombus, tissue protrusion) in $78 \%$ of patients (versus 33\% in matched lesions, $\mathrm{p}<0.001)$ and multiple potential causes in $43 \%$ (versus $3 \%$ in matched lesions, $p<0.001$ ). In addition, most patients with abnormal morphologies also had reduced final lumen dimensions (final lumen $<80 \%$ of reference lumen). In these two previous registries, ${ }^{3}{ }^{13}$ however, none of the patients underwent IVUS studies at the time of stent thrombosis.

\section{Present study}

The present study shows that significant mechanical problems can be identified readily with IVUS in patients with acute stent thrombosis. Severe stent underexpansion was present in most patients and none of them fulfilled the MUSIC criteria for optimal stent deployment. ${ }^{4}$ The presence of an occlusive thrombus was visualised in every patient. Furthermore, stent related dissections, stent malapposition, and significant inflow/outflow disease were common features. All these findings helped in the decision making process involved in device selection (including the requirement for additional stenting) and balloon sizing during the rescue interventions. The findings were also useful in

Table 2 Intravascular ultrasound findings during stent thrombosis

\begin{tabular}{|c|c|c|c|c|c|c|c|c|c|}
\hline Patient & $\begin{array}{l}\text { Stent MLD } \\
(\mathrm{mm})\end{array}$ & $\begin{array}{l}\text { Stent MLA } \\
\left(\mathrm{mm}^{2}\right)\end{array}$ & $\begin{array}{l}\text { Ref LA } \\
\left(\mathrm{mm}^{2}\right)\end{array}$ & Expansion (\%) & Edge Ds & Malapposition & Asymmetry* & I/O disease & MUSIC criteria \\
\hline 1 & 2.1 & 3.9 & 4.6 & 85 & No & No & 0.8 & Yes/No & No \\
\hline 2 & 2.0 & 3.3 & 7.0 & 47 & No & Yes & 0.79 & Yes/Yes & No \\
\hline 3 & 2.3 & 5.4 & 7.6 & 71 & No & Yes & 0.74 & $\mathrm{No} / \mathrm{Yes}$ & No \\
\hline 4 & 3.3 & 8.6 & 11.7 & 73 & No & No & 0.86 & $\mathrm{No} / \mathrm{No}$ & No \\
\hline 5 & 2.3 & 4.2 & 7.5 & 57 & No & No & 0.7 & Yes/Yes & No \\
\hline 6 & 2.2 & 4.1 & 7.0 & 59 & Yes & No & 0.81 & No/Yes & No \\
\hline 7 & 3.1 & 7.8 & 11.2 & 69 & No & Yes & 0.93 & $\mathrm{No} / \mathrm{Yes}$ & No \\
\hline 8 & 2.0 & 3.2 & 5.1 & 63 & Yes & No & 0.94 & Yes/No & No \\
\hline 9 & 3.2 & 8.0 & 9.8 & 81 & No & No & 0.77 & Yes/No & No \\
\hline 10 & 2.7 & 5.7 & 12.9 & 44 & No & Yes & 0.73 & Yes/Yes & No \\
\hline 11 & 3.0 & 8.0 & 9.0 & 88 & No & No & 0.89 & Yes/Yes & No \\
\hline 12 & 2.3 & 4.2 & 12.5 & 34 & No & Not & 0.76 & Yes/Yes & No \\
\hline
\end{tabular}

Thrombus was visualised in all patients. All patients had intravascular ultrasound evidence of calcium at the stented site.

Ds, Dissection; I/O disease, inflow-oufflow disease; MLA, minimum lumen area; Ref LA, reference segment (mean proximal-distal) lumen area.

*Slice with maximum asymmetry of the stent (minimum lumen diameter/maximum lumen diameter); †stent struts protruded $2 \mathrm{~mm}$ from the LAD into the left main stem, partially entrapping the LCX. 

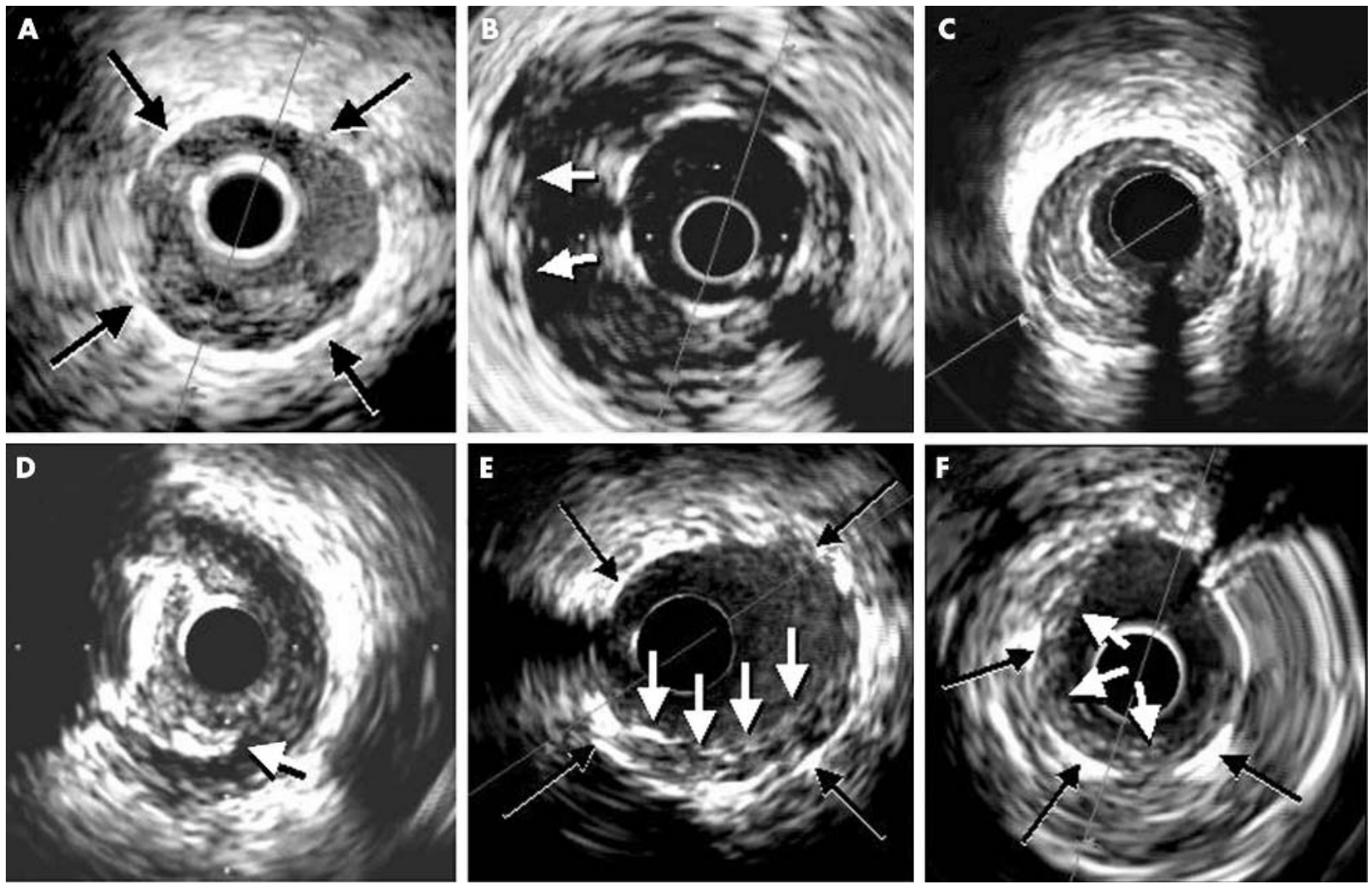

Figure 1 Intravascular ultrasound findings from different patients. Stent thrombosis. (A) A soft material with characteristic speckling is visualised within the stent (black arrows) where the imaging catheter is wedged. (B) Severe malapposition of the stent (at least three stent struts are not placed against the vessel wall (white arrows). (C) Significant plaque burden and reduced residual lumen immediately distal to the stented site. (D) Residual edge dissection (white arrow) emerging from a calcified atherosclerotic plaque. (E, F) Residual lining thrombus (white arrows) within the stent (black arrows) in patients where optimal final results could not be achieved after the intervention.

optimising the final results. Nevertheless, it should be kept in mind that IVUS studies in this setting should be performed only by experienced personnel in well selected cases and always ensuring that this examination is not delaying coronary reperfusion. In patients with ongoing chest pain and persistent ST segment elevation associated with a reduced coronary flow despite the advancement of the guidewire, IVUS studies should be undertaken only after coronary reperfusion has been successfully achieved. In this regard, the selective use of IVUS only after an initial mechanical reperfusion, to guide the procedure and to optimise the final result, also is an attractive option from a more pragmatic stand point.

\section{Non-mechanical factors}

Stent underexpansion is common despite the systematic use of high pressures and modern stent designs, but the incidence of stent thrombosis remains low. ${ }^{1-5}$ Accordingly, a much higher incidence of stent thrombosis would be expected if mild mechanical problems causing a suboptimal stent deployment were the only relevant pathogenetic players accounting for this complication. Nevertheless, the presence of an adverse "thrombogenic milieu" as the result of acute coronary syndromes, inadequate or withheld antithrombotic treatment $^{14}$ or, even more challenging, inter-individual variable response to antithrombotic treatment which may lead to drug resistance (two patients in our study), ${ }^{15-18}$

Table 3 Intravascular ultrasound findings after intervention

\begin{tabular}{|c|c|c|c|c|c|c|c|c|c|c|}
\hline Patient & $\begin{array}{l}\text { Stent } \\
\text { MLD } \\
(\mathrm{mm})\end{array}$ & $\begin{array}{l}\text { Sent MLA } \\
\left(\mathrm{mm}^{2}\right)\end{array}$ & $\begin{array}{l}\text { Ref LA } \\
\left(\mathrm{mm}^{2}\right)\end{array}$ & Expansion (\%) & $\begin{array}{l}\text { Residual } \\
\text { thrombus }\end{array}$ & Edge Ds & Malapposition & Asymmetry & I/O disease & MUSIC criteria \\
\hline 1 & 2.9 & 4.3 & 4.5 & 95 & No & No & No & 0.86 & Yes/No & Yes \\
\hline 2 & 2.5 & 5.3 & 10.3 & 51 & No & No & Yes & 0.85 & No/Yes & No \\
\hline 3 & 2.6 & 5.6 & 7.6 & 74 & No & No & No & 0.76 & No/Yes & No \\
\hline 4 & 3.5 & 10 & 11.0 & 91 & Yes & No & No & 0.85 & $\mathrm{No} / \mathrm{No}$ & Yes \\
\hline 5 & 2.8 & 6.1 & 6.1 & 100 & Yes & No & No & 0.79 & $\mathrm{No} / \mathrm{No}$ & Yes \\
\hline 6 & 2.8 & 5.9 & 5.4 & 109 & Yes & No & No & 0.86 & No/Yes & Yes \\
\hline 7 & 3.2 & 8.3 & 11.2 & 74 & Yes & No & No & 0.85 & No/Yes & No \\
\hline 8 & 2.5 & 4.9 & 5.1 & 96 & Yes & Yes & No & 0.86 & $\mathrm{No} / \mathrm{No}$ & Yes \\
\hline 9 & 3.4 & 9.2 & 11.4 & 81 & Yes & No & No & 0.87 & Yes/No & Yes \\
\hline 10 & 3.1 & 7.7 & 12.9 & 60 & Yes & No & No & 0.88 & Yes/Yes & No \\
\hline 12 & 3.6 & 10.4 & 12.5 & 84 & Yes & No & No & 0.83 & Yes/Yes & Yes \\
\hline
\end{tabular}

Patient 11 did not undergo intravascular ultrasound after the procedure. 
appears to have major implications and should also be ruled out. Although appealing, systematic screening for resistence to platelet inhibition in all patients undergoing coronary stenting is not currently feasible. In this regard it is also of interest that all patients in our series had acute coronary syndromes at the time of initial stent implantation and the potential implications of this finding should be emphasized. ${ }^{2}{ }^{19}$ In a recent postmortem study of 12 patients with fatal "late" stent thrombosis, Farb et al ${ }^{20}$ demonstrated disruption of adjacent vulnerable plaques in two patients and stenting across markedly necrotic, lipid rich plaques with plaque prolapse within the stent struts in two additional patients. Furthermore, although the increasing use of platelet glycoprotein IIb/IIIa inhibitors may dramatically reduce the incidence of acute thrombosis, this would not be expected to influence the rate of subacute stent thrombosis once these drugs are no longer effective. ${ }^{21}$ However, the routine use of these agents in this setting is highly appealing. In addition, although the use of thrombus removal devices alone appears to be an attractive strategy for patients where IVUS has ruled out significant mechanical problems, this approach is still speculative. Finally, whether additional treatments (mechanical or pharmacological) would prove useful in patients with a significant IVUS detected residual burden of "resistant" thrombus at the end of the procedure also remains to be determined.

\section{Clinical implications}

Most patients experiencing stent thrombosis have underexpanded stents, frequently associated with other mechanic abnormalities. Despite a recent meta-analysis ${ }^{22}$ suggesting the potential value of routine IVUS guidance during stent implantation, this strategy is not widely used mainly due to logistic and economic implications. In this regard, our data further emphasises the potential value of IVUS guidance for optimise stent implantation. The extent to which even subtle degrees of stent underexpansion, malapposition, edge tears, or inflow/outflow disease as detected by IVUS contribute to stent thrombosis remains to be fully defined. ${ }^{3}{ }^{13} 2123$ However, the rationale for identifying these potentially reversible mechanical predisposing factors in these pateints is robust, and the use of IVUS for this purpose and to guide repeated interventions is appealing. We suggest that IVUS detected underlying mechanical problems are warning signals that should stimulate further mechanical interventions to optimise the results at the culprit stent, not withstanding a comprehensive search for other non-mechanical predisposing factors for this dreaded complication.

\section{Authors' affiliations}

F Alfonso, A Suárez, D J Angiolillo, M Sabaté, J Escaned, R Moreno, R Hernández, C Bañuelos, C Macaya, Interventional Cardiology Department, Cardiovascular Institute, San Carlos University Hospital, Madrid, Spain

\section{REFERENCES}

1 Hall P, Nakamura S, Maiello L, et al. A randomized comparison of combined ticlopidine and aspirin therapy versus aspirin therapy alone after successful intravascular ultrasound-guided stent implantation. Circulation 1996;93:215-22.

2 Mousa I, di Mario C, Reimers B, et al. Subacute stent thrombosis in the era of intravascular ultrasound-guided coronary stenting without anticoagulation: frequency, predictors and clinical outcome. J Am Coll Cardiol 1997;29:6-12.

3 Uren NG, Schwarzacher SP, Metz JA, et al. Predictors and outcomes of stent thrombosis: an intravascular ultrasound registry. Eur Heart $J$ 2002;23:124-32.

4 de Jaegere $\mathbf{P}$, Mudra $\mathrm{H}$, Figulla $\mathrm{H}$, et al. Intravascular ultrasound-guided optimized stent deployment: immediate and 6-months clinical and angiographic results from the multicenter ultrasound stenting in coronary arteries study (MUSIC study). Eur Heart J 1998;19:1214-23.

5 Bermejo J, Botas J, García E, et al. Mechanisms of residual lumen stenosis after high pressure stent implantation: a quantitative coronary angiography and intravascular ultrasound study. Circulation 1998;98:112-8.

6 Alfonso F, Hernandez R, Goicolea J, et al. Coronary stenting for acute coronary dissection after coronary angioplasty: implications of residual dissection. J Am Coll Cardiol 1994;24:989-95.

7 Alfonso F, Rodriguez P, Phillips $\mathrm{P}$, et al. Clinical and angiographic implications of coronary stenting in thrombus-containing lesions. J Am Coll Cardiol 1997;29:25-733.

8 Mintz GS, Nissen SE, Anderson WD, et al. American College of Cardiology clinical expert consensus document on standards for acquisition, measurement and reporting of intravascular ultrasound studies (IVUS). J Am Coll Cardiol 2001;37:1478-92.

9 Alfonso F, Macaya C, Goicolea J, et al. Determinants of coronary compliance in patients with coronary artery disease: an intravascular ultrasound study. J Am Coll Cardiol 1994;23:879-84.

10 Alfonso F, García P, Pimentel G, et al. Predictors and implications of residual plaque burden after coronary stenting: an intravascular ultrasound study. Am Heart J 2003;145:254-61

11 Hong MK, Park SW, Lee NH, et al. Long-term outcome of minor dissections at the edge of stents detected with intravascular ultrasound. Am J Cardiol 2000;86:791-5.

12 Hoffmann R, Mintz GS, Duissaillant GR, et al. Patterns and mechanisms of instent restenosis: a serial intravascular ultrasound study. Circulation 1996;94:1247-54.

13 Cheneau E, Leborgne L, Mintz A, et al. Predictors of subacute stent thrombosis: results of a systematic intravascular ultrasound study. Circulation 2003; 108:2-5.

14 Kaluza GL, Joshep J, Lee JR, et al. Catastrophic outcomes of noncardiac surgery soon after coronary stenting. J Am Coll Cardiol 2000;35:1288-94.

15 Walter DH, Schächinger V, Elsner M, et al. Platelet glycoprotein Illa polymorphism and risk of coronary stent thrombosis. Lancet 1997;350:1217-9.

16 Jäemo $\mathrm{P}$, Lindahl TL, Fransson SG, et al. Individual variations of platelet inhibition after loading doses of clopidogrel. $J$ Intern Med 2002;252:233-8.

17 Lau WC, Waskell LA, Watkins PB, et al. Atorvastatin reduces the ability of clopidogrel to inhibit platelet aggregation: a new drug-drug interaction. Circulation 2003;107:32-7.

18 Eikelboom JW, Hankey GJ. Aspirin resistance: a new independent predictor of vascular events? J Am Coll Cardiol 2003;41:966-8.

19 Cutlip DE, Baim DS, Kalon KL, et al. Stent thrombosis in the modern era: a pooled analysis of multicenter coronary stent clinical trials. Circulation 2001;103:1967-71.

20 Farb A, Burke AP, Kolodgie FD, et al. Pathologic mechanisms of fatal late coronary stent thrombosis in humans. Circulation 2003;108:1701-6.

21 King SB. Stent Thrombosis: "(ultra) sound the warning". Eur Heart $J$ 2001;23:97-98.

22 Casella G, Klauss V, Ottani F, et al. Impact of intravascular ultrasound-guided stenting on long-term clinical outcome: a meta-analysis comparing ultrasound guided and angiographically guided stenting. Catheter Cardiovasc Interv 2003;59:314-21.

23 Honda Y, Fitzgerald PJ. Stent trombosis. An issue revisited in a changing world. Circulation 2003;108:2-5. 